\title{
ARTICLE OPEN Single microcolony diffusion analysis in Pseudomonas aeruginosa biofilms
}

\author{
Jagadish Sankaran ${ }^{1,2}$, Nicholas J. H. J. Tan ${ }^{3,4}$, Ka Pui But ${ }^{2,5}$, Yehuda Cohen ${ }^{3,4}$, Scott A. Rice $\mathbb{D}^{3,4,6 *}$ and Thorsten Wohland (iD ${ }^{1,2,5 *}$
}

The influence of the biofilm matrix on molecular diffusion is commonly hypothesized to be responsible for emergent characteristics of biofilms such as nutrient trapping, signal accumulation and antibiotic tolerance. Hence quantifying the molecular diffusion coefficient is important to determine whether there is an influence of biofilm microenvironment on the mobility of molecules. Here, we use single plane illumination microscopy fluorescence correlation spectroscopy (SPIM-FCS) to obtain 3D diffusion coefficient maps with micrometre spatial and millisecond temporal resolution of entire Pseudomonas aeruginosa microcolonies. We probed how molecular properties such as size and charge as well as biofilm properties such as microcolony size and depth influence diffusion of fluorescently labelled dextrans inside biofilms. The 2 MDa dextran showed uneven penetration and a reduction in diffusion coefficient suggesting that the biofilm acts as a molecular sieve. Its diffusion coefficient was negatively correlated with the size of the microcolony. Positively charged dextran molecules and positively charged antibiotic tobramycin preferentially partitioned into the biofilm and remained mobile inside the microcolony, albeit with a reduced diffusion coefficient. Lastly, we measured changes of diffusion upon induction of dispersal and detected an increase in diffusion coefficient inside the biofilm before any loss of biomass. Thus, the change in diffusion is a proxy to detect early stages of dispersal. Our work shows that 3D diffusion maps are very sensitive to physiological changes in biofilms, viz. dispersal. However, this study also shows that diffusion, as mediated by the biofilm matrix, does not account for the high level of antibiotic tolerance associated with biofilms.

npj Biofilms and Microbiomes (2019)5:35; https://doi.org/10.1038/s41522-019-0107-4

\section{INTRODUCTION}

Biofilms are aggregates of microbes that adhere to biotic or abiotic surfaces encased in a self-produced matrix of extracellular polymeric substances (EPS). ${ }^{1}$ The proximity of cells embedded in the EPS enable intercellular communication and metabolite exchange that underpins the emergence of properties of biofilms that are distinct from free-living planktonic cells. ${ }^{2}$ Some of these emergent properties include signal accumulation, enhanced horizontal gene exchange, differential access to nutrients through sorption to the EPS and an increased tolerance to antimicrobials ${ }^{3}$ and host immune response. ${ }^{4}$ Biofilm formation increases antimicrobial tolerance by several mechanisms, including an increased transmission of resistance between the different organisms within the biofilm due to high population densities and proximity of cells, ${ }^{5}$ sequestration due to interaction with EPS $^{6}$ and the presence of metabolically inactive persister cells that survive antimicrobial treatment. ${ }^{7}$

Differential access to nutrients leads to altered metabolic activity at different regions of a biofilm. ${ }^{8}$ This leads to physiological heterogeneity at multiple spatial scales. There is considerable diversity not only across different microcolonies within a biofilm but also within the different regions of a single microcolony. The architectural and biochemical heterogeneity arises due to chemical gradients, ${ }^{9,10}$ stochastic gene expression ${ }^{11}$ and genetic variation. ${ }^{12}$ This inherent variation is reflected in the emergent properties that arise upon the conversion of planktonic cells into the biofilm state and can be quantified by measuring the abundance of various molecules, ${ }^{13}$ by measuring gene expression levels ${ }^{14}$ respiratory activity, ${ }^{15}$ stiffness of biofilms ${ }^{16}$ or the diffusion and mobility of molecules within biofilms. ${ }^{17-28}$

The mobility of molecules in biofilms has been quantified using fluorescence recovery after photobleaching, ${ }^{17,18,22,26,28}$ single particle tracking (SPT), ${ }^{27}$ and fluorescence correlation spectroscopy (FCS). ${ }^{19-21,23-25}$ Apart from quantifying the pore size of biofilms ${ }^{24}$ and the mobility of phages in biofilms, ${ }^{20,21}$ FCS studies ${ }^{29}$ have also been used to characterize the role of charge ${ }^{19,25}$ and cell wall hydrophobicity ${ }^{23}$ in determining molecular diffusion in bacterial microcolonies. However, past measurements either determined diffusion only at selected points or were averaged over large volumes of the colonies, thus lacking spatial resolution. To overcome this limitation, we have used here a combination of single plane illumination microscopy (SPIM) and FCS called SPIM$\mathrm{FCS}^{30,31}$ that provides spatially resolved diffusion maps. A typical workflow of SPIM-FCS is shown in Fig. 1a.

SPIM has multiple advantages over the traditionally used confocal microscope. A confocal microscope focuses a laser beam to a small spot and scans this beam over the sample to obtain an image. As the laser beam illuminates all molecules along its path, confocal microscopy requires a pinhole to restrict detection to a small observation volume. Therefore, confocal microscopy sequentially records each point in a sample plane while constantly illuminating the whole sample. This leads to unnecessary photobleaching and phototoxicity. SPIM, on the other hand, illuminates a whole cross-section of the sample using a laser light sheet. An orthogonally placed camera simultaneously records all points. It thus illuminates only those parts of a sample that are recorded and avoids unnecessary photodamage. Hence, in

\footnotetext{
${ }^{1}$ Department of Biological Sciences, National University of Singapore, 117558 Singapore, Singapore. ${ }^{2}$ Centre for Biolmaging Sciences, National University of Singapore, 117557 Singapore, Singapore. ${ }^{3}$ Singapore Centre for Environmental Life Sciences Engineering, Nanyang Technological University, 637551 Singapore, Singapore. ${ }^{4}$ School of Biological Sciences, Nanyang Technological University, 637551 Singapore, Singapore. ${ }^{5}$ Department of Chemistry, National University of Singapore, 117543 Singapore, Singapore. ${ }^{6}$ ithree Institute, University of Technology Sydney, Sydney, Australia. *email: rscott@ntu.edu.sg; twohland@nus.edu.sg
} 
a

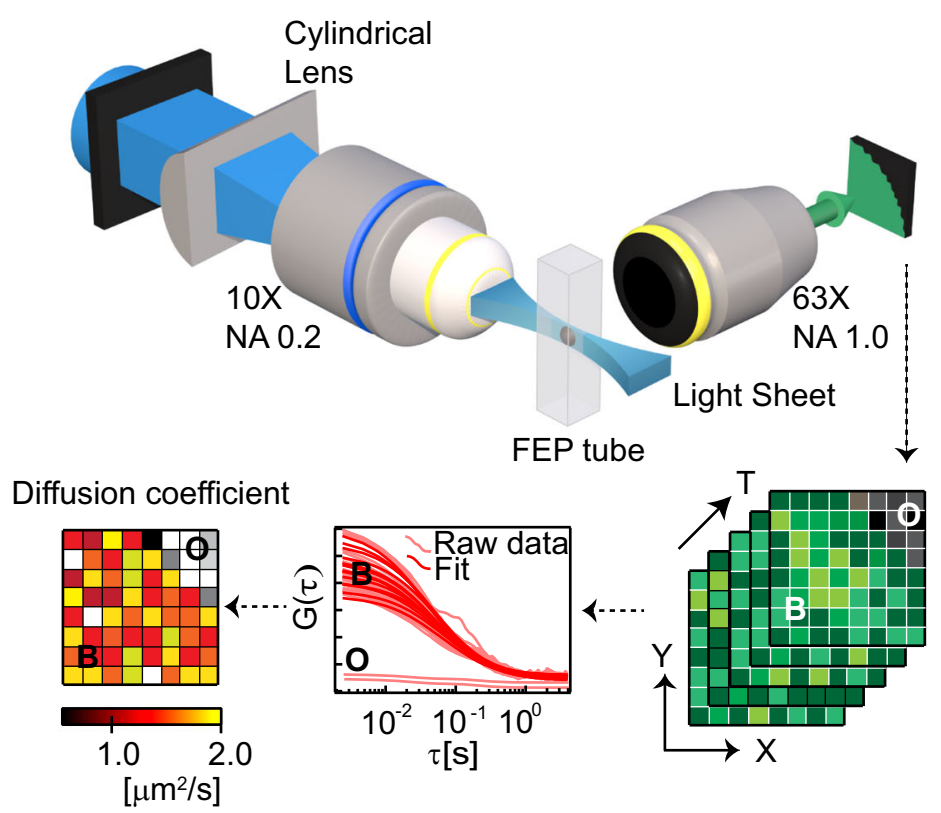

b

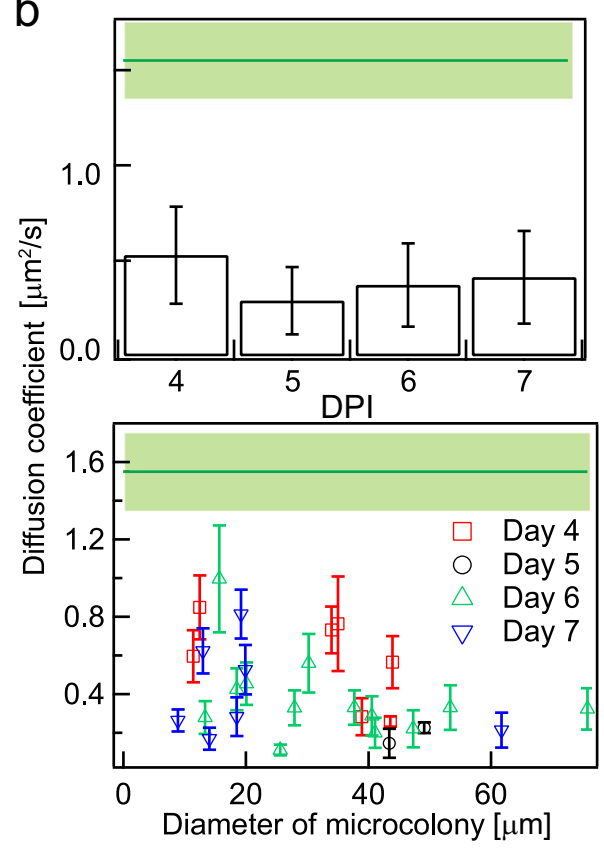

C

d

e
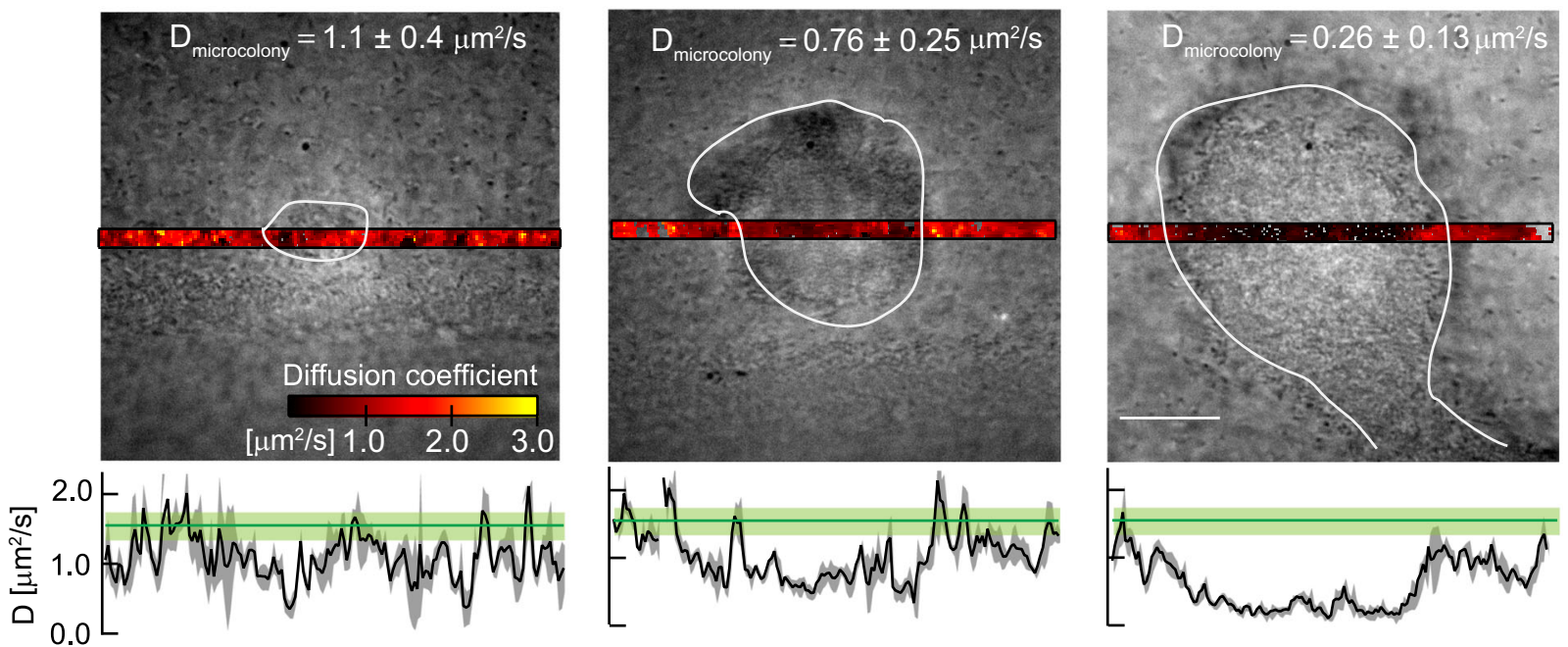

$\tau[\mathrm{s}]$

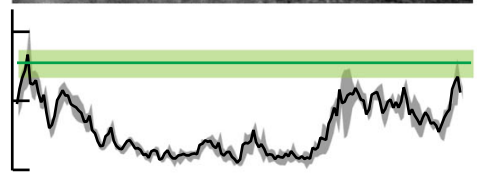

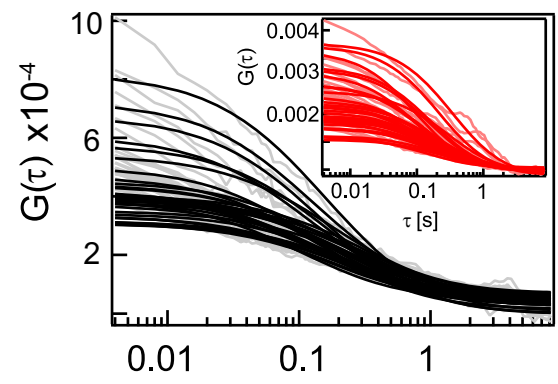

$\tau[\mathbf{s}]$

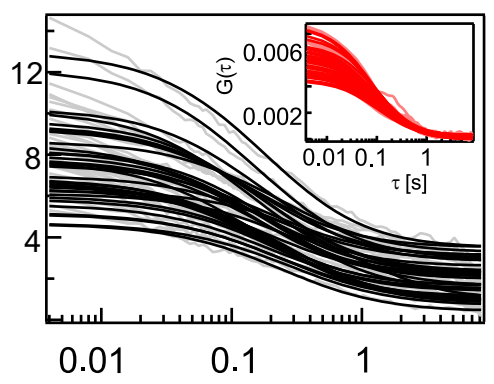

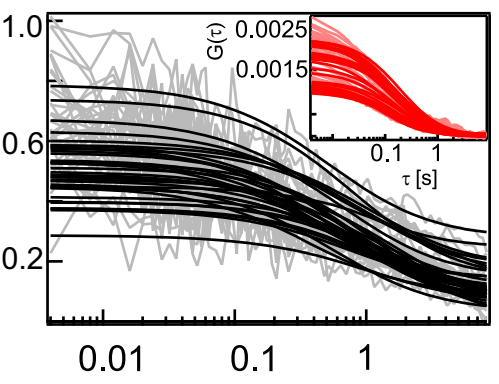

$\tau[\mathrm{s}]$ contrast to confocal microscopy, which requires scanning in all three dimensions to reconstruct an image of the entire microcolony, SPIM requires scanning only along the depth of the microcolony. The combination of SPIM with the fast acquisition of FCS allows for determination of diffusion maps in whole cross- sections of biofilms, which can be sequentially recorded at different depths to provide three-dimensional (3D) diffusion maps of whole colonies. This provides contiguous 3D diffusion profiles that can identify regions of different mobility important in biofilm development. Such diffusion maps provide the ability to 
Fig. 1 Diffusion of 2 MDa dextran inside biofilms: a schematic of SPIM-FCS is shown in a. An EMCCD camera mounted in a light sheet microscope records an image stack. The diffusion coefficients extracted after fitting the calculated autocorrelation functions are displayed as a parametric map. Bright regions in diffusion maps indicate regions of higher mobility when compared to the dim regions, which represent regions of lower mobility. White pixels indicate non-convergence of fits in those regions and hence were not quantified. For the experiments described in this figure, an Andor iXon 897 camera was used as the detector. A plot of the variation in diffusion coefficient of 2 MDa TRITCdextran with days post inoculation (DPI) is shown in $\mathbf{b}$ (top). The same data have been replotted according to colony sizes ( $N=29$ ) in $\mathbf{b}$ (bottom). The solid green line and the light green box in $\mathbf{b}-\mathbf{e}$ is the diffusion coefficient in solution with the corresponding standard deviation. c-e show the wide-field images overlaid with diffusion maps, spatial diffusion profile and autocorrelation curves of three different microcolonies. The boundaries of the microcolony are shown in white contours. The grey area surrounding the black lines are the associated standard deviation in the spatial diffusion profiles. The autocorrelations inside the microcolony are shown in black while the autocorrelations at the outside are shown in red in the inset. The average diffusion coefficient inside the microcolony is displayed in c-e. The error bars in $\mathbf{b}$ show the standard deviation. The scale bar shown in e measures $20 \mu \mathrm{m}$

differentiate gaps in the interstices of neighbouring microcolonies from biomass avoiding erroneous conclusions. ${ }^{32,33}$ SPIM has been used to visualize marine bacteria in their native environment ${ }^{34}$ and to study the population dynamics of artificial ${ }^{35,36}$ and natural $^{37,38}$ communities. $^{39}$ Apart from imaging, SPIM coupled with Transient State Imaging (TRAST) has been used to quantify oxygen abundance in live Pseudomonas aeruginosa biofilms. ${ }^{40}$

In this study, we used SPIM-FCS to investigate the influence of molecular size and charge on diffusion within microcolonies of $P$. aeruginosa of different size, age and stage to determine how the biofilm matrix influences molecular mobility and whether the structure of biofilms could be a reason for the reduced efficacy of antibiotics.

\section{RESULTS}

FCS provides two primary read-outs. The first is the width of the autocorrelation function (ACF) from which the diffusion coefficient can be determined. The second is the amplitude of the ACF that is related to the average number of particles seen in the observation volume of a pixel and is thus a measure of concentration. The relation between amplitude and concentration is complex however, and depends on the signal-to-noise ratio as will be discussed later. Here, we use these two parameters to characterize mobility within and structure of the biofilm matrices.

First, we determined the accuracy and precision of SPIM-FCS for diffusion coefficient measurements for dextrans of molecular weights in the range of $4 \mathrm{kDa}$ to $2 \mathrm{MDa}$ and compared those with measurements made using confocal FCS (Supplementary Fig. 1 and Supplementary Notes 1). We next performed SPIM-FCS measurements in alginate beads using fluorescently labelled dextrans of different size and charge to provide a baseline for biofilm measurements (Supplementary Figs 2-7, Supplementary Notes 2-4). Alginate, a component of EPS, can be easily formed into beads and provides a homogeneous environment to test SPIM-FCS. TRITC-dextran molecules of 20 and $150 \mathrm{kDa}$ penetrated into alginate beads and exhibited a retardation in diffusion inside the alginate bead, although diffusion was uniform throughout the beads. In contrast, while the $2 \mathrm{MDa}$ TRITC-dextran penetrated into alginate beads, it showed considerable variation in diffusion coefficient at different positions.

To disentangle size and charge effects, we therefore conducted measurements with $2 \mathrm{MDa}$ TRITC-dextran to investigate sieving effects in $P$. aeruginosa biofilms, while we used $150 \mathrm{kDa}$ dextrans of different charge to investigate the influence of charge on penetration and diffusion. Images of representative $P$. aeruginosa microcolonies fluorescently stained with Con-A-AlexaFluor 488 are shown in Supplementary Fig. 8.

Diffusion of 2 MDa dextran inside biofilms

TRITC-labelled $2 \mathrm{MDa}$ neutral dextran was added to $P$. aeruginosa biofilms. For biofilms grown for 4-7 days, there was a fourfold reduction in the diffusion coefficient within the biofilm when compared to that in solution (Fig. 1b, top).

We observed a negative correlation between the diffusion coefficient and microcolony size (Fig. 1b, bottom). The Pearson's linear correlation coefficient was found to be $-0.38(N=29)$. Due to the heterogeneity in colony sizes on any given day, we did not detect a statistically significant difference in diffusion coefficient across days post inoculation (Fig. 1b, top, one-way ANOVA, $p=0.13$ ). Small colonies (diameter $<\sim 40 \mu \mathrm{m}, N=19$ ) had a mean diffusion coefficient of $0.49 \pm 0.25 \mu \mathrm{m}^{2} \mathrm{~s}^{-1}$, while for larger colonies (diameter $>\sim 40 \mu \mathrm{m}, N=10$ ) the mean diffusion coefficient was $0.28 \pm 0.12 \mu \mathrm{m}^{2} \mathrm{~s}^{-1}$. Examples of individual colonies ranging from 20 to $70 \mu \mathrm{m}$ are shown in Fig. 1c, d. While small microcolonies showed uniform diffusion profiles (Fig. 1c, d), microcolonies of $\sim 70 \mu \mathrm{m}$ diameter, diffusion was reduced in the centre of the large colonies, shown as a trough in the diffusion profile (Fig. 1e).

In addition to the changes in diffusion, the amplitude of the ACFs decreased by about a factor 5 for the smaller colonies (Fig. $1 c$, d) when comparing the exterior to the interior of the biofilm and almost a factor of 20 for the large colony (Fig. 1e). The correlation curves inside the biofilm were also noisier than those on the outside. The reduction in amplitude and in the signal-tonoise ratio suggests that the concentration inside the biofilm is lower than that of the outside (as described in Supplementary notes S4). This demonstrates that there is a concentration gradient of the 2 MDa TRITC-dextran from the outside to the inside. While qualitatively correct, a quantitative estimation of the concentration cannot be obtained currently due to the lack of methods to estimate the noise levels within individual microcolonies.

\section{D profiles of diffusion coefficients of neutral molecules}

Given the change in diffusion based on microcolony diameter for $2 \mathrm{MDa}$ TRITC-dextran, we next investigated the relationship between the diffusion coefficient and microcolony depths by analysing diffusion of $2 \mathrm{MDa}$ TRITC-dextran in 11 colonies of varying diameters $(20-40 \mu \mathrm{m})$. Overall, we saw a change in the signal-to-noise ratio of the ACFs with depth (Fig. 2a-c, top). There was also an increase in heterogeneity in diffusion maps at 15 and $40 \mu \mathrm{m}$ when compared to $0 \mu \mathrm{m}$ (Fig. 2a-c, bottom, d). The heterogeneity was quantified by the coefficient of variation (COV ratio of the standard deviation to the mean) of the diffusion coefficient that increases with depth (Fig. 2e). The increased COV was manifested as a wider distribution of diffusion coefficients as can be seen from the diffusion coefficient histograms (Fig. 2f) and is a result of the lower signal-to-noise ratio for these measurements. This implies a decrease in concentration (see Supplementary Note 4) with depth, establishing a molecular gradient.

While the colonies in Fig. 2 and Supplementary Fig. 12 are $\sim 30 \mu \mathrm{m}$ thick, the colony in Supplementary Fig. 11 is $\sim 14 \mu \mathrm{m}$ thick. Colonies shown in Fig. 2 and Supplementary Fig. 11 showed considerable changes in COV with depth, while the colony shown in Supplementary Fig. 12 had very little variation in diffusion 

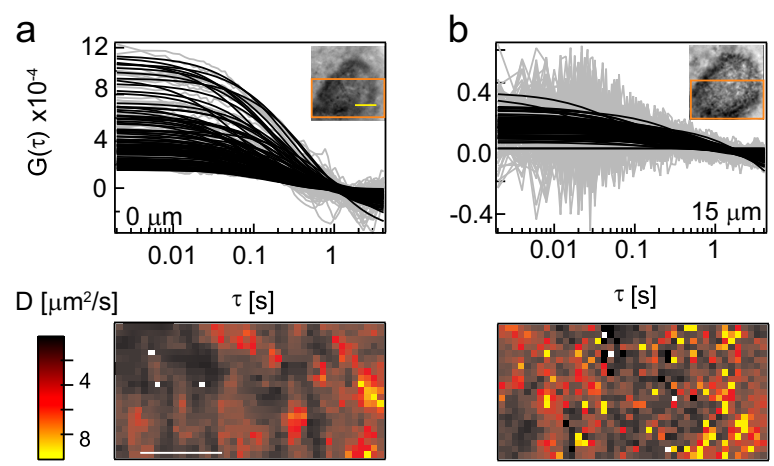

$\tau[\mathrm{s}]$
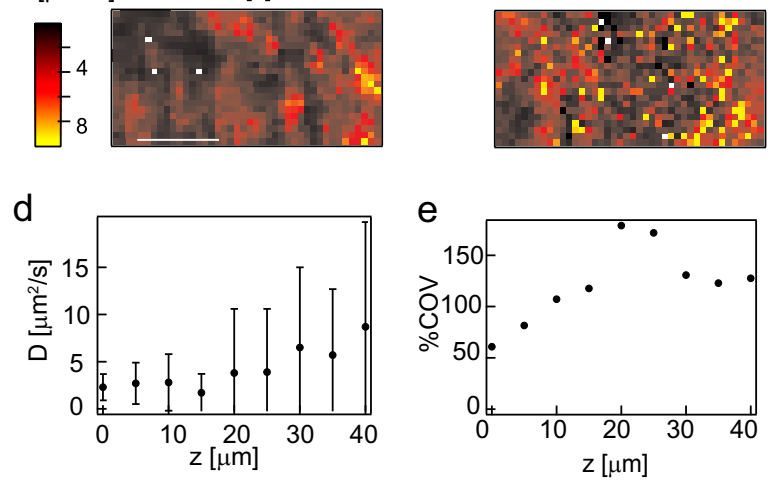
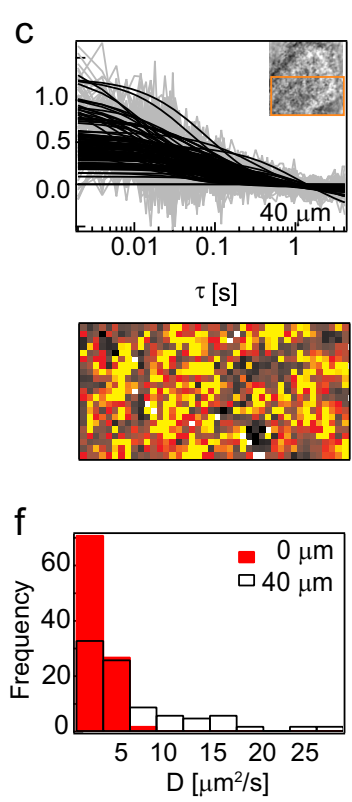

Fig. 2 3D profiles of diffusion coefficients of neutral molecules: a-c show autocorrelation functions, diffusion maps and wide-field images at three different depths of the biofilm that has a thickness of $40 \mu \mathrm{m}$. The diffusion map is that of the region shown in orange in the inset of a -c. In the autocorrelation functions, the raw data are shown in grey while the fitted data are shown in black. The scale bar in yellow in the inset and the diffusion map in $\mathbf{a}$ is $10 \mu \mathrm{m}$. $\mathbf{d}$ is a plot of the average diffusion coefficient of $2 \mathrm{MDa}$ TRITC-dextran with depth of the biofilm whose wide-field images are shown in Supplementary Fig. 10. The coefficient of variation, which is the ratio of the standard deviation to the mean, is shown in e. $\mathbf{f}$ shows the distribution of diffusion coefficients for the solution and at the base of the biofilm. Supplementary Figure 10 is an accompanying figure to this main figure. The raw and fitted autocorrelation functions along with the diffusion maps at all measured depths is shown in Supplementary Fig. 10. The error bars in $\mathbf{d}$ show the standard deviation

coefficient, with consistent high signal-to-noise ratio, low COV and narrow distributions of the diffusion coefficient. This implies considerable heterogeneity in depth profiles for the diffusion coefficient and the molecular concentration gradient was not related to the size of the biofilm (as shown in Fig. 2, Supplementary Figs 11 and 12) even when the average diffusion coefficient within the colonies was correlated to overall size.

\section{Diffusion of charged molecules in biofilms}

Next, FITC-labelled $150 \mathrm{kDa}$ dextran coupled with positive (diethyl aminoethyl-DEAE; zeta potential $=34.3 \pm 3.8 \mathrm{mV}$ ) or negative charges (carboxy methyl-CM; zeta potential $=-23.5 \pm 3.2 \mathrm{mV}$ ) were added to biofilms to study how charge affects the diffusion of molecules in microcolonies. When the positively charged $150 \mathrm{kDa}$ FITC-DEAE-dextran (DEAE-dex) was used, it preferentially partitioned into the biofilm (Fig. 3a, inset bottom). The intensity profile along the red line in Fig. 3a shows that the intensity is higher in the biofilm when compared to the exterior. In contrast, the untreated $150 \mathrm{kDa}$ FITC-dextran (zeta potential $=-5 \pm 3 \mathrm{mV}$ ) and $150 \mathrm{kDa}$ FITC-CM-dextran did not preferentially partition into the biofilm (Fig. 3b, c, insets) and the intensities were similar on the outside and inside of the biofilms.

The intensity ratio of the interior of the microcolonies $(N=3)$ to that of the exterior was $3.2 \pm 1.0,0.9 \pm 0.1$ and $0.8 \pm 0.2$ for the positively charged, negatively charged and neutral molecules, respectively. In addition, the ACF of $150 \mathrm{kDa}$ FITC-DEAE-dextran had a better signal-to-noise ratio compared to the negative and neutral molecules (Fig. 3a-c). Collectively, these two metrics indicate that the positively charged molecules penetrated with the highest efficacy of the three types of molecules.

The ACF of all three dextrans showed two different diffusion patterns both in solution and in the biofilm and were fitted to a two-component diffusion model (Eq. 3). The slowly diffusing population had diffusion coefficients less than $1 \mu \mathrm{m}^{2} \mathrm{~s}^{-1}$, while the fast diffusing population had diffusion coefficient larger than
$5 \mu \mathrm{m}^{2} \mathrm{~s}^{-1}$. The diffusion coefficients and their fractions are provided in Supplementary Table 7. The presence of two diffusing populations indicates that a certain proportion of probe molecules exist as aggregates in solution and in the biofilm, while the remainder of the probe is present as single molecules.

Only the $150 \mathrm{kDa}$ FITC-DEAE-dextran exhibited a statistically significant reduction $(N=5, p=0.04)$ in the diffusion coefficient of the fast moving population inside the biofilm $\left(6 \pm 4.5 \mu \mathrm{m}^{2} \mathrm{~s}^{-1}\right)$ when compared to solution $\left(15.9 \pm 4.9 \mu \mathrm{m}^{2} \mathrm{~s}^{-1}\right)$. The diffusion coefficient of the slow moving population and the fraction of slow moving population was not significantly different between the biofilm and the solution.

3D profile of diffusion coefficients of positively charged molecule Although positively charged molecules penetrated with highest efficiency, the localization and dynamics of DEAE-dextran at different depths of a microcolony was not homogeneous. One specific example is shown in Fig. 4 and Supplementary Fig. 13. As quantified in Supplementary Table 7 earlier, the particles exhibited a two-component diffusion pattern. Inside the microcolony, the heterogeneity in the diffusion map of the faster component increased with depth (Fig. 4a). The heterogeneity in diffusion coefficients was manifested as an increased standard deviation associated with the estimates of diffusion coefficient (Fig. 4a).

At deeper sections of the microcolony ( $25 \mu \mathrm{m}$ shown in Fig. $4 \mathrm{c}$ and $20-30 \mu \mathrm{m}$ shown in Supplementary Fig. 13), the fluorescent image had three distinct regions, a dim outside, a bright peripheral interior and a dim central interior region. This shows that even though the positively charged molecule had a higher concentration within the biofilm when compared to the exterior, its distribution is not uniform throughout the biofilm.

Similar to the fluorescence intensity, the diffusion maps (25 $\mu \mathrm{m}$ shown in Fig. 4d and 20-30 $\mu \mathrm{m}$ shown in Supplementary Fig. 13) displayed three regions, a heterogeneous exterior, homogeneous peripheral interior and a heterogeneous central interior. A 


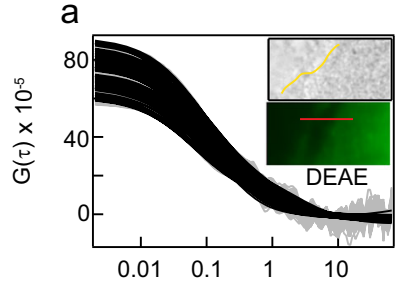

$\tau[\mathrm{s}]$
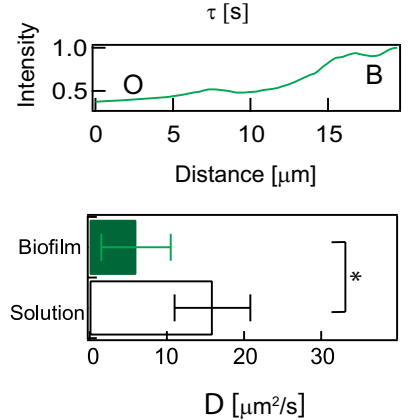

b

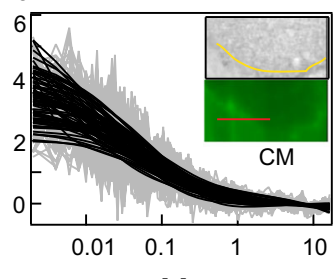

$\tau[\mathrm{s}]$
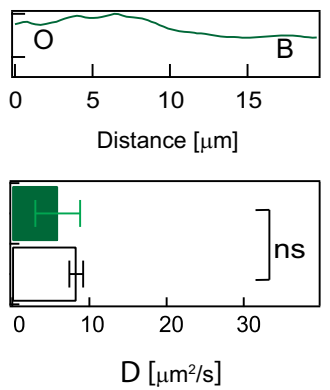

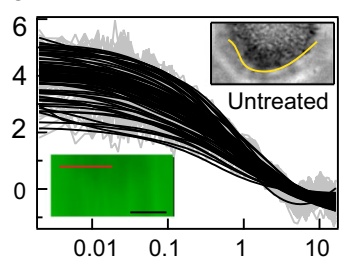

$\tau[\mathrm{s}]$
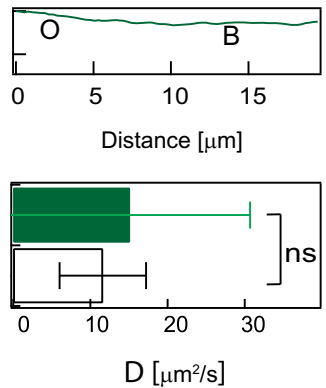

Fig. 3 Diffusion of charged molecules in biofilms: a-c show the wide-field image, fluorescence image and autocorrelation curves when $150 \mathrm{kDa}$ FITC-DEAE-dextran, $150 \mathrm{kDa}$ FITC-CM-dextran and untreated $150 \mathrm{kDa}$ dextran molecules were added to biofilms. The contour of the biofilm is shown in yellow in the wide-field images. The intensity profile along the red line shown in the fluorescence images are shown in the profiles below the autocorrelation curve. O refers to outside while B refers to inside the biofilm. The bar charts show the diffusion coefficients of the fast moving population of positive $(N=5)$, negative $(N=3)$ and neutral $(N=8)$ molecules inside the biofilm and in solution. Only the $150 \mathrm{kDa}$ FITC-DEAE-dextran exhibited a statistically significant reduction $(p=0.04)$ in the diffusion coefficient of the fast moving population inside the biofilm when compared to solution. The raw data and the fitted data are shown in grey and black respectively in a-c. Individual autocorrelation functions in a representative area of 100 pixels are shown in a-c. The significance levels were evaluated using a two-sided Wilcoxon rank test. The error bars show the standard deviation. The scale bar in the inset of c measures $10 \mu \mathrm{m}$

comparison of Fig. 4c, d shows that bright fluorescent regions (marked I-B in Fig. 4c) correspond to the homogeneous regions based on diffusion coefficient and dim fluorescent regions (marked I-D in Fig. 4c) exhibited greater heterogeneity in their diffusion profiles.

The fraction of the slow moving particles in the deep interior of the biofilm increased beyond $20 \mu \mathrm{m}$ (Fig. 4a, inset). The fraction of slow particles was the lowest in the periphery of the biofilm at $25 \mu \mathrm{m}$ (Fig. 4e). The slowly diffusing particles may result from strong interactions with the EPS leading to an increased fraction of slow moving particles in the central interior region of the biofilm.

\section{SPIM-FCS measurements of diffusion of labelled antibiotics}

Given that DEAE-dextran preferentially partitioned into the biofilm, we then tested whether a positively charged aminoglycoside antibiotic tobramycin, ${ }^{41}$ exhibited the same behaviour. Similar to DEAE-dextran, positively charged, TRITC-labelled tobramycin penetrated into the biofilms and preferentially partitioned into the biomass (Fig. 5a, inset).

The intensity profile across the biofilm border clearly shows increased intensity inside the biofilm compared to the exterior (Fig. 5). There was also a 15-fold statistically significant reduction $(N=11 ; p=0.007)$ in the diffusion coefficient of fluorescently labelled tobramycin from solution $\left(15.3 \pm 7.0 \mu \mathrm{m}^{2} \mathrm{~s}^{-1}\right)$ to the interior of the biofilm $\left(0.9 \pm 0.3 \mu \mathrm{m}^{2} \mathrm{~s}^{-1}\right)$ (Fig. 5a). Similar to tobramycin, a neutral fluoroquinolone ciprofloxacin, ${ }^{42}$ fluorescently labelled with Cy5, also accumulated in the biomass and exhibited a 26-fold statistically significant reduction $(N=8 ; p=$ $0.006)$ in diffusion coefficient from solution $\left(33.4 \pm 8.1 \mu \mathrm{m}^{2} \mathrm{~s}^{-1}\right)$ to the biofilm interior $\left(1.3 \pm 0.7 \mu \mathrm{m}^{2} \mathrm{~s}^{-1}\right)$ (Fig. 5b).

The effect of dispersal of $P$. aeruginosa biofilms on molecular diffusion

Next, we probed changes in diffusion coefficient of 2 MDa TRITCdextran for approximately $8 \mathrm{~h}$ as the microcolony was undergoing induced dispersal. The intracellular level of c-di-GMP regulates the transition from biofilm to planktonic phenotype. The cellular concentration of bis- $\left(3^{\prime}-5^{\prime}\right)$-cyclic dimeric guanosine monophosphate (c-di-GMP) is dynamically controlled by the opposing activities of multiple diguanylate cyclases (formation of c-di-GMP) and phosphodiesterases (degradation of c-di-GMP).

In the $P$. aeruginosa pBAD yhjH strain, a phosphodiesterase gene was cloned with an arabinose inducible promoter ${ }^{43,44}$ to induce biofilm dispersal. The microcolony was imaged for $8 \mathrm{~h}$ after the addition of the inducing molecule-arabinose. The diffusion coefficient inside the microcolony $7.5 \mathrm{~h}$ post induction increased twofold when compared to that in the solution at $z=8 \mu \mathrm{m}$ (Fig. $6 \mathrm{~d})$. The periphery between the exterior of the microcolony and the interior of the microcolony is discernible in the diffusion maps at the bottom most plane after at least $4 \mathrm{~h}$ of induction (Fig. 6). A similar trend was also seen in another microcolony (Supplementary Fig. 14) in which the diffusion coefficient inside the microcolony was higher than that of the outside at $7.5 \mathrm{~h}$ post induction in the bottom most plane at $z=15 \mu \mathrm{m}$. However, only 2 out of 10 colonies imaged exhibited this behaviour within $8 \mathrm{~h}$. The other eight colonies did not show an increase in diffusion coefficient inside the microcolony post induction. This suggests that SPIM-FCS is able to monitor changes in physicochemical properties of the biofilm during dispersal before the detection of biomass loss.

\section{DISCUSSION}

In this study, we used SPIM-FCS to measure 3D diffusion profiles of molecules of different size and charge in biofilms of various sizes, providing insights into biofilm structure from attachment to dispersal. SPIM-FCS is an ideal tool for this task, due to the reduced photobleaching and photodamage and increased speed of measurements compared to confocal FCS. Performing diffusion studies at the single microcolony level allowed quantification and visualization of the heterogeneity of diffusion and structure in biofilms. 
a

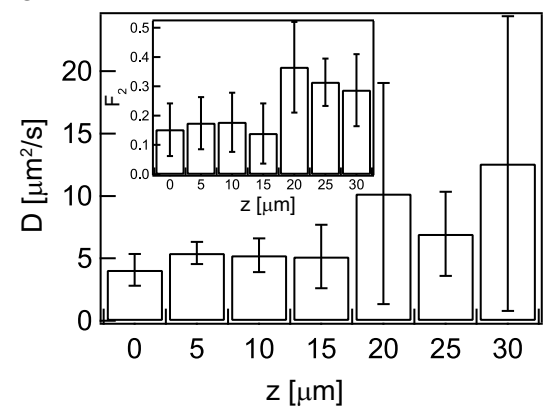

b

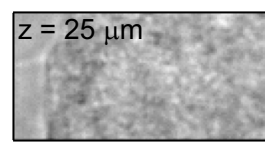

d

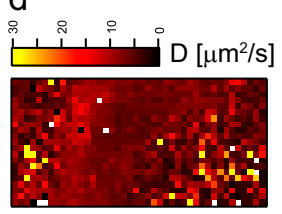

C

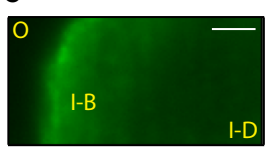

e

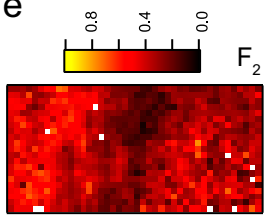

$\mathrm{f}$

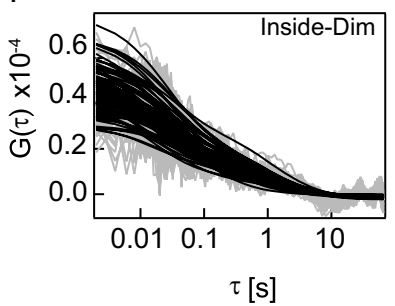

g

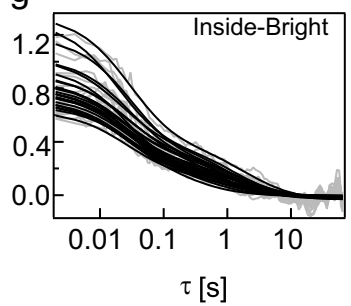

$\mathrm{h}$

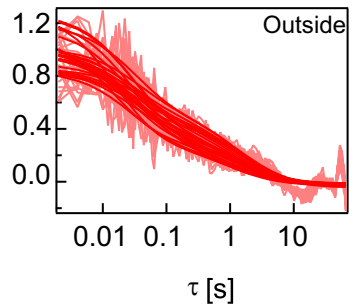

Fig. 4 3D profile of diffusion coefficients of positively charged molecule: $\mathbf{a}$ is a plot of the variation of the diffusion coefficient inside the biofilm with the $z$ position when $150 \mathrm{kDa}$ DEAE-dextran was added to the biofilm. The inset shows the fraction of the slow moving particle. Only one plane at $z=25 \mu \mathrm{m}$ is shown here, while the entire $z$ stack is shown in Supplementary Fig. 13. b and $\mathbf{c}$ are the wide field and fluorescent images, respectively. O refers to outside, I-B refers to "inside-bright", I-D refers to "inside-dim". The faster diffusion coefficient and the fraction of the slow particle $\left(F_{2}\right)$ is shown in $\mathbf{d}$ and $\mathbf{e}$, respectively. The autocorrelation curves of the dim region in the interior of the biofilm $(I-D)$ in the fluorescence image is shown in $\mathbf{f}$. The autocorrelation curves of bright interior (I-B) region of the biofilm in the fluorescence image is shown in $\mathbf{g}$. $\mathbf{h}$ is a plot of the autocorrelation curves at the outside of the biofilm. The error bars in a show the standard deviation. The scale bar in c measures $10 \mu \mathrm{m}$

The EPS matrix of biofilms acts as a molecular sieve ${ }^{3}$ and restricts the penetration of molecules into the biofilm larger than its pore size. Previous estimates of pore size ranged from 500 to $1000^{45}$ and $125 \mathrm{~nm}^{27,46}$ by different methods for $P$. aeruginosa PAO1, the same strain used here. In the case of Pseudomonas fluorescens, loose flocs had an effective pore size of $50 \mathrm{~nm}$, which decreased to $10 \mathrm{~nm}$ for dense biofilms. ${ }^{24}$ In this study, molecules up to hydrodynamic radius of $27 \mathrm{~nm}$ penetrate into the biofilm, setting a lower limit for the pore size and diffuse slower in biofilms when compared to free solution. Even though there is variability in the beginning of microcolony formation, a reduction in porosity occurs as they increase in size. Despite the fact that $2 \mathrm{MDa}$ TRITCdextran penetrated into the biofilm, there was a concentration difference between the outside and the inside of the biofilms. The reduction in diffusion coefficient of probes exogenously added to biofilms is best described by the obstruction model of diffusion in hydrogels. ${ }^{47,48}$ In this model, the biofilm acts as a sieve and solutes with sizes smaller than the pore size of the biofilm can diffuse into the biofilm. The mobility inside the biofilm is directly related to the void fraction of the biofilm and inversely related to the tortuosity of the biofilm. This may be because as the size of the molecule increases, there is hindered diffusion ${ }^{49}$ or an entanglement with the polymeric material that constitutes the EPS. ${ }^{50,51}$

We parametrized the geometry of a 3D microcolony as two independent parameters, lateral diameter and axial depth. While there was no statistically significant difference in diffusion coefficient across days post inoculation, there was a correlation between the diffusion coefficient and the diameter of the microcolony in which it was measured. Small colonies had a higher average diffusion coefficient than larger colonies. The heterogeneity in diffusion coefficients of colonies with a diameter of 20-30 $\mu \mathrm{m}$ suggests that the crosslinking of polymers in the small colonies may differ sufficiently to alter diffusion of these molecules. This suggests that the matrix composition of the microcolonies may change over time leading to changes in crosslinking and porosity. This is also consistent with AFM data, showing that the Young's modulus of early stage biofilms is less than that of older, larger microcolonies. ${ }^{52}$ The correlation between diffusion coefficient and size of a microcolony also suggests that microcolony size is a more robust metric than age when comparing and characterizing biofilms.

While colony size, rather than age, was a better predictor of diffusion properties, colonies of the same size can have very different 3D structures and this may also influence diffusion behaviours. In a subset of colonies $(20 \%)$ we found clear changes with depth, manifested as decreased signal-to-noise ratios, larger COVs and broader distributions for the measured diffusion coefficients. Our current explanation is that as the depth increases, the crosslinking of the polymers in the EPS increases, leading to a decrease in porosity and a more heterogeneous environment, thus leading to reduced penetration of the probe to deeper regions of the biofilm. In the other $80 \%$ of microcolonies, the probes penetrated evenly throughout the colony. This suggests that in a given population of microcolonies, there is variability in the crosslinking of EPS with depth of the microcolony, independent of the diameter.

The self-produced matrix of EPS in P. aeruginosa consists of positively charged (polysaccharide-Pel, ${ }^{53}$ ) negatively charged (eDNA, ${ }^{54}$ alginate, ${ }^{55}$ filamentous phage $\mathrm{Pf}^{56}$ and neutral molecules (polysaccharide-Psl. ${ }^{54}$ ) The heterogeneity in the distribution of the $150 \mathrm{kDa}$ FITC-DEAE-dextran in different regions of the biofilm is an indication of a non-uniform charge distribution which might arise due to the variation in the spatial distribution of charged molecules within a biofilm. ${ }^{57}$

We hypothesize that the decreased mobility of the positively charged DEAE-dextran molecules might be due to either replacement of cationic molecules in EPS or electrostatic interactions with negatively charged molecules in the EPS. Positively charged exopolysaccharide-Pel is a cross-linker found in the EPS. The decreased mobility of the DEAE-dextran molecule might be due to the replacement of Pel in the EPS network by 

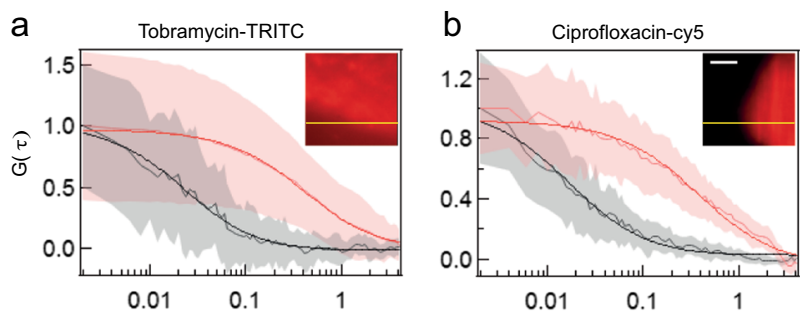

$\tau[\mathrm{s}]$
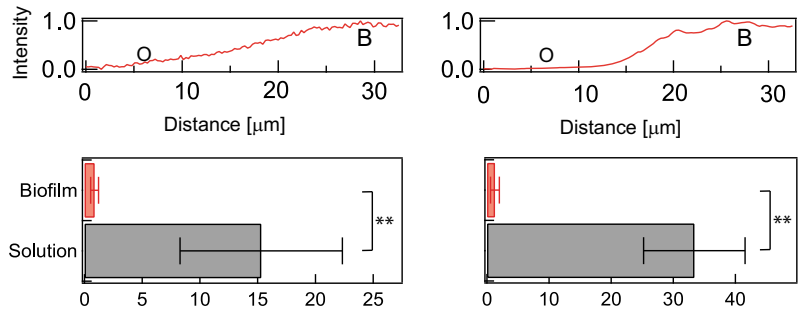

$\mathrm{D}\left[\mu \mathrm{m}^{2} / \mathrm{s}\right]$

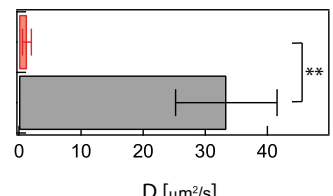

Fig. 5 SPIM-FCS measurements of diffusion of labelled antibiotics: The average autocorrelation curve of fluorescently labelled tobramycin and ciprofloxacin is shown in red in $\mathbf{a}$ and $\mathbf{b}$, respectively. The light red shading around the curve is the error over an average of 100 pixels. The curve in black in $\mathbf{a}$ and $\mathbf{b}$ is the average autocorrelation curve inside the biofilm. The inset shows a fluorescence image of a biofilm to which fluorescently labelled tobramycin and ciprofloxacin have been added. The intensity profile along the yellow line in the insets is shown below the autocorrelation plots. $\mathbf{b}$ denotes the biofilm. Bar charts show the diffusion coefficients of antibiotics within the biofilm and in solution. The measurements in solutions are an average of three measurements each. In the case of biofilms, these are an average of 11 and 8 microcolonies for tobramycin $(p=0.007)$ and ciprofloxacin $(p=$ $0.006)$, respectively. In the autocorrelation plots, the raw data are shown in grey and light red while the fitted data are shown in black and red. The significance levels were determined using a two-sided Wilcoxon rank test. The error bars in $\mathbf{a}$ and $\mathbf{b}$ show the standard deviation. The scale bar in the inset of $\mathbf{b}$ measures $10 \mu \mathrm{m}$

DEAE-dextran. Apart from replacement, the reduction in mobility of positively charged molecules might also be due to interactions with the negatively charged components of EPS matrix such as filamentous phage Pf4 or eDNA. Cationic molecules bind and cross-link Pf4 phages ${ }^{56}$ and such binding will lead to a reduction in mobility. eDNA chelates cations as they pass into the biofilm matrix $^{59}$ through electrostatic interactions. ${ }^{58}$

Similar to observations in this study, using SPT, it was shown that positively charged molecules accumulate within the biomass. ${ }^{27}$ In contrast to $P$. aeruginosa, cationic molecules did not penetrate biofilms of Lactococcus lactis or Stenotrophomonas maltophilia ${ }^{19}$ and this may be a consequence of the biofilm matrices being composed of different biopolymers for the various bacteria.

To monitor bacteria in different physiological states during the biofilm life-cycle, we performed SPIM-FCS on a strain undergoing induced dispersal. After inducing dispersal, there was an increase in the diffusion coefficient inside the biofilm that was even higher than diffusion as measured in solution. We hypothesize that this may be due to the fluidic activity during the seeding dispersal ${ }^{59}$ and would be consistent with a breakdown of the matrix and increased motility of the bacteria, which is required for bacteria to escape the matrix during dispersal.

Eighty per cent of the colonies did not show an increase in diffusion coefficient. We speculate that the dispersal process was not induced in these colonies. The low efficacy of dispersal could be due to the use of arabinose induction media containing glucose. Glucose is not completely exhausted in the medium during the induction and hence the cells are not physiologically prepared for dispersal. On the contrary, the cells experiencing starvation are more prone to dispersal due to changes in c-di-GMP. Determination of the timeframe in which dispersal is induced will be useful for the fields of clinical microbiology, internal medicine and pharmacology as therapeutic solutions have advocated the use of dispersal agents in addition to antibiotic treatment ${ }^{60}$ along with time-dependent dosage of antimicrobials. ${ }^{61}$

Similar to observations by Walters et al., ${ }^{33}$ both ciprofloxacin and tobramycin penetrated into $P$. aeruginosa biofilms. Ciprofloxacin and tobramycin accumulated in the $P$. aeruginosa biofilm and exhibited reduced diffusion compared to solution. The reduction of the diffusion coefficient of tobramycin is in agreement with previous observations of reduced tobramycin diffusion by a disc diffusion assay in the presence of alginate ${ }^{62}$ and can also be explained by the interactions with the EPS of $P$. aeruginosa. ${ }^{63}$ The estimates of the increase in concentration and decrease in diffusivity inside the biofilm for tobramycin is useful when modelling the bacterial biofilm as a reaction-diffusion system. ${ }^{64}$ The increase in concentration of tobramycin inside seaweed alginate beads was earlier explained by theoretical studies using a reaction-diffusion model with nonspecific power law binding to polyanion matrix components. ${ }^{65}$ Our measurements showed a 1.5 -fold increase in intensity of labelled tobramycin inside the biofilm when compared to the exterior.

Results from Landry et al. ${ }^{66}$ suggest that the efficiency of penetration of tobramycin into "flat, homogeneous" biofilms is higher than penetration into biofilms characterized with "large cell aggregates". In the case of "fully matured biofilms with large cell aggregates", tobramycin was sequestered in the periphery of the microcolonies. ${ }^{67}$ We observed penetration of tobramycin into the biofilm microcolony and did not see sequestration of tobramycin in the periphery since the penetration experiments were performed on flat biofilms, which did not have mushroom shaped structures, but rather, the microcolonies appear as flattened mounds of cells.

Previous reports and our own results have shown that the biofilm does not represent a diffusion barrier for antibiotics ${ }^{26,28}$ in $P$. aeruginosa biofilms despite a reduction in diffusion coefficient. Together, our results suggest that diffusion limitations do not contribute to the observed tolerance in clinically relevant biofilms for antimicrobials. This suggests that other mechanisms that convey antibiotic tolerance in biofilms, ${ }^{64,68}$ such as physiological differentiation, play a more important role in biofilm tolerance to antibiotics than the extracellular matrix.

It is well known that biofilms exhibit physiological, architectural and biochemical heterogeneity. Our results suggest that there is also considerable heterogeneity in the diffusion of molecules in biofilms. It remains to be tested whether the physiological heterogeneity in biofilms is a cause or an effect of heterogeneity in diffusion. The mobility of molecules diffusing inside biofilms obtained in this study is not only useful in gaining an understanding of the fundamental principles governing diffusion in a heterogeneous biofilm system but also has utilization in the pharmacokinetic modelling of drugs and in mass transfer modelling in bioreactors for wastewater treatment. Information about the effects of molecular properties on their diffusion in biofilms along with the effects of biofilm physiology on molecular diffusion is invaluable in the selection and design of agents that can effectively penetrate the biofilm matrix to reach the target site of action. For example, the results from this study could be used to design improved drug delivery systems. Based on the signal-tonoise ratio of the raw data of the autocorrelation curves and imaging studies using the three charged molecules, we suggest that a carrier with an outer, positively charged surface would have the highest penetration compared to neutral or negatively charged molecules. Quantification of the diffusion coefficient of molecules along with their variation in living biofilms will aid in efficient strategizing of the kinetics upon the addition of biofilm removal agents in clinical and industrial settings. 

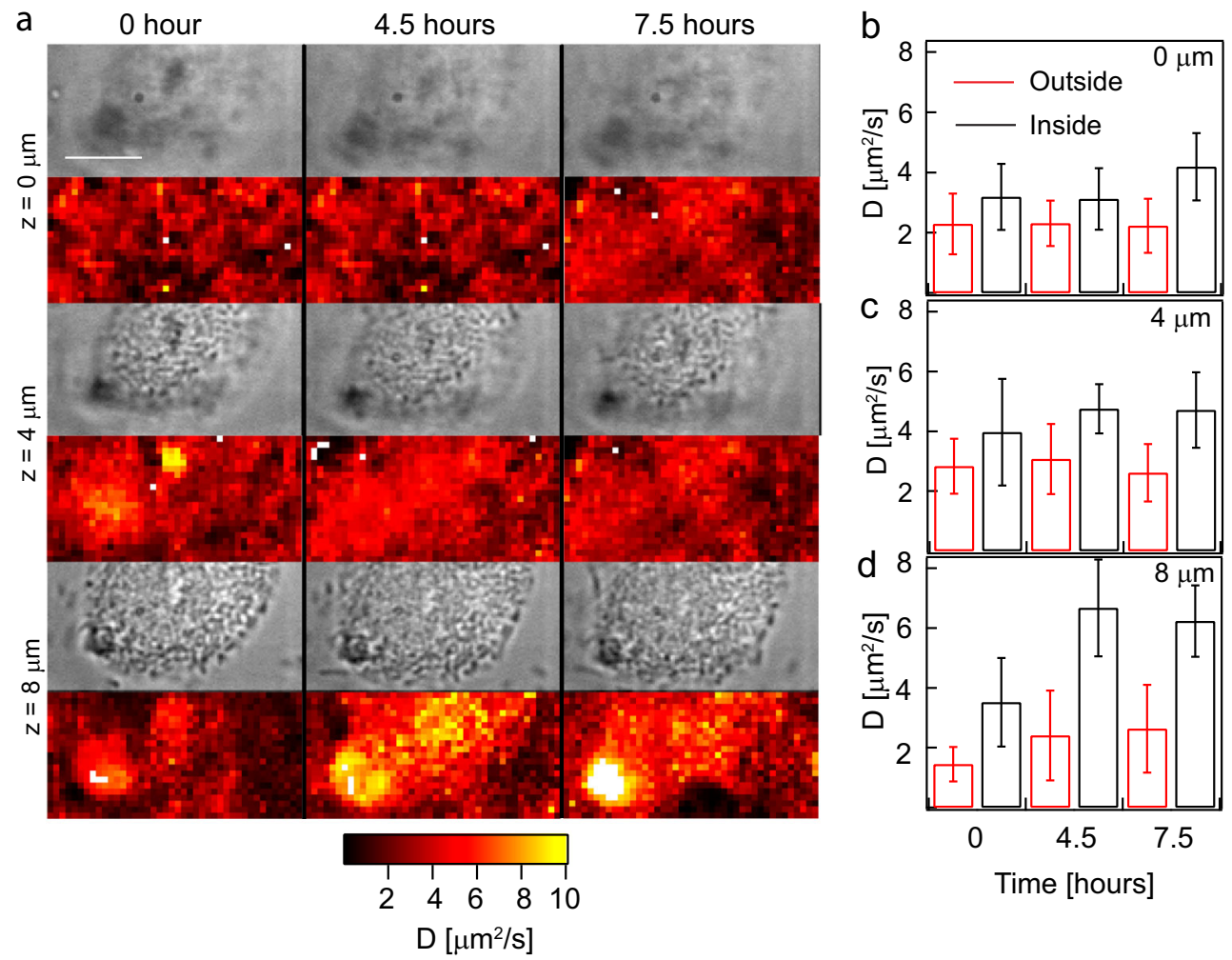

Fig. 6 The effect of dispersal of $P$. aeruginosa biofilms on molecular diffusion: $\mathbf{a}$ is a montage of the $z$ stacks and time-lapse of the wide field and parametric diffusion maps at various times post induction using $1 \%$ arabinose of $P$. aeruginosa PA01 eGFP pBAD yhjH biofilm grown in ABGC gentamycin media. The diffusion coefficients of $2 \mathrm{MDa}$ dextran measured at different depths and at different time points were quantified and shown in $\mathbf{b}-\mathbf{d}$. The error bars in $\mathbf{b}-\mathbf{d}$ show the standard deviation. The scale bar in a measures $10 \mu \mathrm{m}$

\section{METHODS}

P. aeruginosa PA01 pBAD yhjH, ${ }^{43}$ PA01 wild type, PA01 eGFP and PA01 PBAD yhjH eGFP have been used in this study. Single colonies were inoculated into $10 \mathrm{~mL}$ Luria-Bertani (LB) medium $\left(10 \mathrm{~g} \mathrm{~L}^{-1}\right.$ tryptone, $5 \mathrm{~g} \mathrm{~L}^{-1}$ yeast extract, $10 \mathrm{~g} \mathrm{~L}^{-1}$ sodium chloride) and incubated overnight at $25^{\circ} \mathrm{C}$ to prepare the inoculum. For plasmid maintenance in $P$. aeruginosa PA01 pBAD yhjH, LB was supplemented with $30 \mu \mathrm{g} \mathrm{mL}^{-1}$ gentamycin.

TRITC-dextrans of various molecular weights ( $2 \mathrm{MDa}, 500,150,70,40,20$ and $4 \mathrm{kDa}$ ), diethyl aminoethyl, carboxy methyl dextran and neutral $150 \mathrm{kDa}$ FITC-dextran were obtained from TdB Consultancy (Uppsala, Sweden), and dissolved in water to prepare stock solutions. TRITC-labelled tobramycin and cy5-labelled ciprofloxacin were purchased from Bioconjugate Technology Company (Scottsdale, AZ, USA). Except for the 2 MDa conjugates, SPIMFCS experiments on alginate beads were done with $100 \mathrm{nM}$ TRITC-dextran. In the case of FITC-dextran, $250 \mathrm{nM}$ and $1 \mu \mathrm{M}$ were used for alginate beads and biofilms. In the case of $2 \mathrm{MDa}, 10 \mathrm{nM}$ was used for alginate beads and biofilms for confocal and SPIM-FCS. In the case of confocal FCS, 30-250 nM was used $(4,20,40,70,150$ and $500 \mathrm{kDa})$. A concentration of $100 \mathrm{nM}$ of labelled tobramycin and ciprofloxacin was used for the experiments.

\section{Generation of PA01 eGFP pBAD yhjH strain}

The eGFP-expressing strain carries a gentamycin resistance cassette as part of the transposon used to introduce the gfp gene. Therefore, this gentamycin cassette was first removed from the chromosome using the FLIP recombinase system and selected for gentamycin sensitivity. Subsequently, a gentamycin-sensitive isolate was transformed with the pBAD yhjH plasmid and selected on gentamycin plates.

\section{Biofilm growth conditions}

P. aeruginosa PA01 eGFP pBAD yhjH was grown in $\mathrm{AB}^{69}$ minimal medium containing $15.1 \mathrm{mM}\left(\mathrm{NH}_{4}\right)_{2} \mathrm{SO}_{4}, 33.7 \mathrm{mM} \mathrm{Na}_{2} \mathrm{HPO}_{4}, 22 \mathrm{mM} \mathrm{KH}_{2} \mathrm{PO}_{4}, 0.05 \mathrm{mM}$ $\mathrm{NaCl}, 1 \mathrm{mM} \mathrm{MgCl}, 100 \mu \mathrm{M} \mathrm{CaCl}$ and $1 \mu \mathrm{M} \mathrm{FeCl}$. The medium was supplemented with $0.2 \%$ glucose $(\mathrm{G})$ and $0.2 \%$ casamino acids (C). This medium is referred to as $A B G C$ medium. For dispersal experiments, for $\mathrm{PBAD}$ yhjH plasmid maintenance, $30 \mu \mathrm{g} \mathrm{mL}^{-1}$ gentamycin was added to the medium (ABGC gen media). Media was pumped at a rate of $5 \mathrm{~mL} \mathrm{~h}^{-1}$ using a peristaltic pump (Cole Parmer Masterflex (Vernon Hills, IL, USA)—flow speed at 8) into the flow cell through fluorinated ethylene propylene (FEP) tubing.

A single colony was inoculated into $10 \mathrm{~mL}$ medium and incubated at $25^{\circ} \mathrm{C}$ and $200 \mathrm{rpm}$ overnight. In the case of PA01 wild type (used in all experiments except those described in Fig. 6 and Supplementary Fig. 14), the overnight culture was diluted to an optical density $\left(\mathrm{OD}_{600}\right)$ of 0.3 before injection into flow setup. Flow was stopped before inoculation and resumed $1 \mathrm{~h}$ after inoculation. In the case of PA01 eGFP pBAD yhjH, the overnight culture was subcultured till the $\mathrm{OD}_{600}$ reached 0.1 and then injected as inoculum into the flow chamber. The $\mathrm{OD}_{600}$ was chosen to be 0.1 since live wide-field microscopy showed a reduction in biomass visually after arabinose induction.

\section{SPIM-FCS}

Biofilms were grown in $3 \mathrm{~cm}$ long square profile $\mathrm{FEP}^{70}$ tubes (nominal size $2 \mathrm{~mm} \times 2 \mathrm{~mm}$; Adtech, UK) in minimal medium. FEP was chosen because the refractive index of the material matches that of the water used as immersion medium for the objective and because the tube was sturdy enough to withhold shear stresses due to the flow in the flow chamber. The biofilms grew on all four sides of the tube. The biofilms growing on the side closest to the detection objective were used for imaging and spectroscopy. The position of light sheet is fixed in the microscope, hence different regions of the biofilm can be illuminated by moving the sample relative to the light sheet. Data from different microcolonies from different flow experiments were compiled to estimate diffusion coefficients.

FEP tubes were removed from the flow cells and one end was sealed with glue. Colonies were imaged using Carl Zeiss commercial light sheet microscope Z.1 (referred to as light sheet 1). Two different EMCCD cameras (Andor, Belfast, UK) controlled using Andor Solis software were used as the detectors (Andor 860-referred to as camera 1 and Andor 897, referred to as camera 2). Measurements for Fig. 1 and Supplementary Fig. 8 were performed in camera 2 while the rest of experiments involving biofilms in this manuscript were performed with camera 1. Unless otherwise stated in the figure legend, Andor iXon 860 was used for the detection.

A laser power of $1-3 \mathrm{~mW}$ was used for illumination. LSFM $\times 10$ / NA0.2 served as the illumination objective while a WPlan-Apochromat $\times 63$ / 
NA1.0 was used as the detection objective. After blocking the excitation light, the detected fluorescence was filtered using SBSLP510, LP565 and 660DF50 filters for 488,561 and $638 \mathrm{~nm}$ lasers, respectively. Laser blanking was switched off and a macro was used to create a static light sheet.

The metadata showed that the scaling $X$ and $Y$ were $72 \mathrm{~nm}$. This corresponded to $\times 90$ magnification. A frame length of 50,000 with $0.002 \mathrm{~s}$ exposure time was used throughout the paper except for the data in Fig. 1. A frame length of 100,000 frames with $0.004 \mathrm{~s}$ exposure time was used for the experiments in Fig. 1. Data analysis was performed using an ImageJ plugin called Imaging FCS 1.47 (ref. ${ }^{71}$ ). The fitting model used to determine the diffusion coefficient is provided in the manual of the software. Polynomial order 4 bleach correction was used. The data were binned $4 \times 4$ in the case of Supplementary Fig. 1 . The rest of the data is binned $3 \times 3$. The fitting model used was

$G(\tau)=\frac{1}{N} \frac{g(\tau)}{g(0)}$,

where

$$
\begin{gathered}
g(\tau)=\left(\frac{\sqrt{4 D \tau+\omega_{x y}^{2}}}{a \sqrt{\pi}}\left(\mathrm{e}^{-\frac{a^{2}}{4 D \tau+\omega_{x y}^{2}}}-1\right)+\operatorname{Erf}\left(\frac{a}{\sqrt{4 D \tau+\omega_{x y}^{2}}}\right)\right)^{2} \\
\times\left(1+\frac{4 D \tau}{\omega_{z}^{2}}\right)^{-\frac{1}{2}}+G_{\infty},
\end{gathered}
$$

where $a$ is the pixel size, $\tau$ is the lag time, $N$ is the number of particles, $D$ is the diffusion coefficient, $\omega_{x y}$ is the PSF in the xy direction while $\omega_{z}$ is the thickness of the light sheet. The procedure to determine $\omega_{x y}$ and $\omega_{z}$ is described in Supplementary Methods. The experimental values are also tabulated in the same section. In the case of two components, the fitting model is given by

$G(\tau)=\frac{\left(1-F_{2}\right) \frac{g_{1}(\tau)}{g_{1}(0)}+F_{2} \frac{g_{2}(\tau)}{g_{2}(0)}}{N}+G_{\infty}$,

where $F_{2}$ is the fraction of the slow moving particle obtained from curve fitting. $D_{1}$ and $D_{2}$ are the diffusion coefficients of the fast and the slow moving particles respectively.

\section{Zeta potential measurements}

The zeta potential of untreated, DEAE and CM dextran were measured using Malvern Zetasizer Nano (Worcestershire, UK). A concentration of $10 \mathrm{mg} \mathrm{mL}^{-1}$ was used for all the three probes. The probes were dissolved in DI water for the measurement.

\section{Statistical tests}

All the statistical tests were performed in Igor Pro $^{\odot}$ (Wavemetrics, OR, USA).

\section{Reporting summary}

Further information on research design is available in the Nature Research Reporting Summary linked to this article.

\section{DATA AVAILABILITY}

The datasets generated during the current study are available from the corresponding author on reasonable request.

Received: 23 January 2019; Accepted: 4 October 2019; Published online: 08 November 2019

\section{REFERENCES}

1. Hall-Stoodley, L., Costerton, J. W. \& Stoodley, P. Bacterial biofilms: from the natural environment to infectious diseases. Nat. Rev. Microbiol. 2, 95-108 (2004).

2. Flemming, H. C. et al. Biofilms: an emergent form of bacterial life. Nat. Rev. Microbiol. 14, 563-575 (2016).

3. Flemming, H. C. \& Wingender, J. The biofilm matrix. Nat. Rev. Microbiol. 8, 623-633 (2010)

4. Maunders, E. \& Welch, M. Matrix exopolysaccharides; the sticky side of biofilm formation. FEMS Microbiol. Lett. 364, fnx120-fnx120 (2017).

5. Balcázar, J. L., Subirats, J. \& Borrego, C. M. The role of biofilms as environmental reservoirs of antibiotic resistance. Front. Microbiol. 6, 1216 (2015).
6. Shan, T. B. et al. The extracellular matrix protects Pseudomonas aeruginosa biofilms by limiting the penetration of tobramycin. Environ. Microbiol. 15, 2865-2878 (2013).

7. Lewis, K. Multidrug tolerance of biofilms and persister cells. Curr. Top. Microbiol. Immunol. 322, 107-131 (2008).

8. Stewart, P. S. \& Franklin, M. J. Physiological heterogeneity in biofilms. Nat. Rev. Microbiol. 6, 199-210 (2008).

9. Debeer, D., Stoodley, P., Roe, F. \& Lewandowski, Z. Effects of biofilm structures on oxygen distribution and mass-transport. Biotechnol. Bioeng. 43, 1131-1138 (1994).

10. Lim, C. P., Mai, P. N. Q., Roizman Sade, D., Lam, Y. C. \& Cohen, Y. Biofilm development of an opportunistic model bacterium analysed at high spatiotemporal resolution in the framework of a precise flow cell. NPJ Biofilms Microbiomes 2, 16023 (2016).

11. Chai, Y., Chu, F., Kolter, R. \& Losick, R. Bistability and biofilm formation in Bacillus subtilis. Mol. Microbiol. 67, 254-263 (2008).

12. Kirisits, M. J., Prost, L., Starkey, M. \& Parsek, M. R. Characterization of colony morphology variants isolated from Pseudomonas aeruginosa biofilms. Appl. Environ. Microbiol. 71, 4809-4821 (2005).

13. Neu, T., Swerhone, G. D. \& Lawrence, J. R. Assessment of lectin-binding analysis for in situ detection of glycoconjugates in biofilm systems. Microbiology 147, 299-313 (2001).

14. Huang, C.-T., Xu, K. D., McFeters, G. A. \& Stewart, P. S. Spatial patterns of alkaline phosphatase expression within bacterial colonies and biofilms in response to phosphate starvation. Appl. Environ. Microbiol. 64, 1526-1531 (1998).

15. Huang, C. T., Yu, F. P., McFeters, G. A. \& Stewart, P. S. Nonuniform spatial patterns of respiratory activity within biofilms during disinfection. Appl. Environ. Microbiol. 61, 2252-2256 (1995).

16. Karampatzakis, A. et al. Probing the internal micromechanical properties of Pseudomonas aeruginosa biofilms by Brillouin imaging. NPJ Biofilms Microbiomes 3, 20 (2017).

17. Birmingham, J. J., Hughes, N. P. \& Treloar, R. Diffusion and binding measurements within oral biofilms using fluorescence photobleaching recovery methods. Philos. Trans. R. Soc. Lond. B Biol. Sci. 350, 325-343 (1995).

18. Bryers, J. D. \& Drummond, F. Local macromolecule diffusion coefficients in structurally non-uniform bacterial biofilms using fluorescence recovery after photobleaching (FRAP). Biotechnol. Bioeng. 60, 462-473 (1998).

19. Guiot, E. et al. Heterogeneity of diffusion inside microbial biofilms determined by fluorescence correlation spectroscopy under two-photon excitation. Photochem. Photobiol. 75, 570-578 (2002).

20. Lacroix-Gueu, P., Briandet, R., Lévêque-Fort, S., Bellon-Fontaine, M.-N. \& FontaineAupart, M.-P. In situ measurements of viral particles diffusion inside mucoid biofilms. C. R. Biol. 328, 1065-1072 (2005).

21. Briandet, R. et al. Fluorescence correlation spectroscopy to study diffusion and reaction of bacteriophages inside biofilms. Appl. Environ. Microbiol. 74 2135-2143 (2008).

22. Waharte, F., Steenkeste, K., Briandet, R. \& Fontaine-Aupart, M. P. Diffusion measurements inside biofilms by image-based fluorescence recovery after photobleaching (FRAP) analysis with a commercial confocal laser scanning microscope. Appl. Environ. Microbiol. 76, 5860-5869 (2010).

23. Habimana, O. et al. Diffusion of nanoparticles in biofilms is altered by bacterial cell wall hydrophobicity. Appl. Environ. Microbiol. 77, 367-368 (2011).

24. Peulen, T.-O. \& Wilkinson, K. J. Diffusion of nanoparticles in a biofilm. Environ. Sci. Technol. 45, 3367-3373 (2011).

25. Zhang, Z., Nadezhina, E. \& Wilkinson, K. J. Quantifying diffusion in a biofilm of Streptococcus mutans. Antimicrob. Agents Chemother. 55, 1075-1081 (2011).

26. Daddi Oubekka, S., Briandet, R., Fontaine-Aupart, M. P. \& Steenkeste, K. Correlative time-resolved fluorescence microscopy to assess antibiotic diffusion-reaction in biofilms. Antimicrob. Agents Chemother. 56, 3349-3358 (2012).

27. Forier, K. et al. Transport of nanoparticles in cystic fibrosis sputum and bacterial biofilms by single-particle tracking microscopy. Nanomedicine 8, 935-949 (2013).

28. Boudjemaa, R. et al. New insight into daptomycin bioavailability and localization in Staphylococcus aureus biofilms by dynamic fluorescence imaging. Antimicrob. Agents Chemother. 60, 4983-4990 (2016).

29. Sankaran, J., Karampatzakis, A., Rice, S. A. \& Wohland, T. Quantitative imaging and spectroscopic technologies for microbiology. FEMS Microbiol. Lett. 365, 12 (2018).

30. Wohland, T., Shi, X., Sankaran, J. \& Stelzer, E. H. K. Single Plane Illumination Fluorescence Correlation Spectroscopy (SPIM-FCS) probes inhomogeneous threedimensional environments. Opt. Express 18, 10627-10641 (2010).

31. Krieger, J. W., Singh, A. P., Garbe, C. S., Wohland, T. \& Langowski, J. Dual-color fluorescence cross-correlation spectroscopy on a single plane illumination microscope (SPIM-FCCS). Opt. Express 22, 2358-2375 (2014).

32. Davies, D. Understanding biofilm resistance to antibacterial agents. Nat. Rev. Drug Discov. 2, 114 (2003)

33. Walters, M. C. 3rd, Roe, F., Bugnicourt, A., Franklin, M. J. \& Stewart, P. S. Contributions of antibiotic penetration, oxygen limitation, and low metabolic activity 
to tolerance of Pseudomonas aeruginosa biofilms to ciprofloxacin and tobramycin. Antimicrob. Agents Chemother. 47, 317-323 (2003).

34. Fuchs, E., Jaffe, J. S., Long, R. A. \& Azam, F. Thin laser light sheet microscope for microbial oceanography. Opt. Express 10, 145-154 (2002).

35. Hekstra, D. R., Cocco, S., Monasson, R. \& Leibler, S. Trend and fluctuations: analysis and design of population dynamics measurements in replicate ecosystems. Phys. Rev. E 88, 062714 (2013).

36. Hekstra, D. R. \& Leibler, S. Contingency and statistical laws in replicate microbial closed ecosystems. Cell 149, 1164-1173 (2012).

37. Jemielita, M. et al. Spatial and temporal features of the growth of a bacterial species colonizing the zebrafish gut. Mbio 5, e01751-14 (2014).

38. Wiles, T. J. et al. Host gut motility promotes competitive exclusion within a model intestinal microbiota. PLoS Biol. 14, e1002517 (2016).

39. Parthasarathy, R. Monitoring microbial communities using light sheet fluorescence microscopy. Curr. Opin. Microbiol. 43, 31-37 (2018).

40. Karampatzakis, A. et al. Measurement of oxygen concentrations in bacterial biofilms using transient state monitoring by single plane illumination microscopy. Biomed. Phys. Eng. Express 3, 035020 (2017).

41. Hermann, T. Aminoglycoside antibiotics: old drugs and new therapeutic approaches. Cell. Mol. Life Sci. 64, 1841-1852 (2007).

42. Cavet, M. E., West, M. \& Simmons, N. L. Fluoroquinolone (ciprofloxacin) secretion by human intestinal epithelial (Caco-2) cells. Br. J. Pharmacol. 121, 1567-1578 (1997).

43. Chua, S. L. et al. Bis-(3'-5')-cyclic dimeric GMP regulates antimicrobial peptide resistance in Pseudomonas aeruginosa. Antimicrob. Agents Chemother. 57, 2066-2075 (2013).

44. Chua, S. L. et al. In vitro and in vivo generation and characterization of Pseudomonas aeruginosa biofilm-dispersed cells via c-di-GMP manipulation. Nat. Protoc. 10, 1165 (2015).

45. Chew, S. C. et al. Dynamic remodeling of microbial biofilms by functionally distinct exopolysaccharides. mBio 5, e01536-14 (2014).

46. Teirlinck, E., Samal, S. K., Coenye, T. \& Braeckmans, K. in Functionalized Nanomaterials for the Management of Microbial Infection (eds. Boukherroub, R., Szunerits, S. \& Drider, D.) 49-76 (Elsevier, 2017).

47. Fiorentino, S. M. et al. in Handbook of Membrane Separations 436-481 (CRC Press, 2015).

48. Amsden, B. Solute diffusion within hydrogels. mechanisms and models. Macromolecules 31, 8382-8395 (1998).

49. Yeon, W. C., Kannan, B., Wohland, T. \& Ng, V. Colloidal crystals from surfacetension-assisted self-assembly: a novel matrix for single-molecule experiments. Langmuir 24, 12142-12149 (2008).

50. Wöll, D. Fluorescence correlation spectroscopy in polymer science. RSC Adv. 4, 2447-2465 (2014)

51. Liu, R., Gao, X., Adams, J. \& Oppermann, W. A fluorescence correlation spectroscopy study on the self-diffusion of polystyrene chains in dilute and semidilute solution. Macromolecules 38, 8845-8849 (2005).

52. Kundukad, B. et al. Mechanical properties of the superficial biofilm layer determine the architecture of biofilms. Soft Matter 12, 5718-5726 (2016).

53. Franklin, M. J., Nivens, D. E., Weadge, J. T. \& Howell, P. L. Biosynthesis of the Pseudomonas aeruginosa extracellular polysaccharides, alginate, Pel, and Psl. Front. Microbiol. 2, 167-167 (2011).

54. Mulcahy, H., Charron-Mazenod, L. \& Lewenza, S. Extracellular DNA chelates cations and induces antibiotic resistance in Pseudomonas aeruginosa biofilms. PLoS Pathog. 4, e1000213 (2008).

55. Jones, C. J. \& Wozniak, D. J. Psl produced by mucoid Pseudomonas aeruginosa contributes to the establishment of biofilms and immune evasion. mBio 8, e00864-00817 (2017).

56. Patrick, R. et al. Filamentous Bacteriophage Promote Biofilm Assembly and Function. Cell Host Microbe. 18, 549-559 (2015).

57. Allesen-Holm, M. et al. A characterization of DNA release in Pseudomonas aeruginosa cultures and biofilms. Mol. Microbiol. 59, 1114-1128 (2006).

58. Purdy Drew, K. R., Sanders, L. K., Culumber, Z. W., Zribi, O. \& Wong, G. C. L. Cationic amphiphiles increase activity of aminoglycoside antibiotic tobramycin in the presence of airway polyelectrolytes. J. Am. Chem. Soc. 131, 486-493 (2009).

59. Kaplan, J. B. Biofilm dispersal: mechanisms, clinical implications, and potential therapeutic uses. J. Dent. Res. 89, 205-218 (2010).

60. Fleming, D. \& Rumbaugh, K. P. Approaches to dispersing medical biofilms. Microorganisms 5, 15 (2017).

61. Roizman, D., Vidaillac, C., Givskov, M. \& Yang, L. In-vitro evaluation of biofilm dispersal as a therapeutic strategy to restore antimicrobial efficacy. Antimicrob. Agents Chemother. 61, e01088-17 (2017).

62. Gordon, C. A., Hodges, N. A. \& Marriott, C. Antibiotic interaction and diffusion through alginate and exopolysaccharide of cystic fibrosis-derived Pseudomonas aeruginosa. J. Antimicrob. Chemother. 22, 667-674 (1988).
63. Nichols, W. W., Dorrington, S. M., Slack, M. P. \& Walmsley, H. L. Inhibition of tobramycin diffusion by binding to alginate. Antimicrob. Agents Chemother. 32 , 518-523 (1988).

64. Stewart, P. S. et al. Reaction-diffusion theory explains hypoxia and heterogeneous growth within microbial biofilms associated with chronic infections. Npj Biofilms Microbiomes 2, 16012 (2016).

65. Cao, B. et al. Diffusion retardation by binding of tobramycin in an alginate biofilm model. PLOS ONE 11, e0153616 (2016).

66. Landry, R. M., An, D., Hupp, J. T., Singh, P. K. \& Parsek, M. R. Mucin-Pseudomonas aeruginosa interactions promote biofilm formation and antibiotic resistance. Mol. Microbiol. 59, 142-151 (2006).

67. Tseng, B. S. et al. The extracellular matrix protects Pseudomonas aeruginosa biofilms by limiting the penetration of tobramycin. Environ. Microbiol. 15, 2865-2878 (2013).

68. Stewart, P. S. \& William Costerton, J. Antibiotic resistance of bacteria in biofilms. Lancet 358, 135-138 (2001).

69. Clark, D. J. \& Maaløe, O. DNA replication and the division cycle in Escherichia coli. J. Mol. Biol. 23, 99-112 (1967).

70. Kaufmann, A., Mickoleit, M., Weber, M. \& Huisken, J. Multilayer mounting enables long-term imaging of zebrafish development in a light sheet microscope. Development 139, 3242-3247 (2012).

71. Sankaran, J., Shi, X., Ho, L. Y., Stelzer, E. H. \& Wohland, T. ImFCS: a software for imaging FCS data analysis and visualization. Opt. Express 18, 25468-25481 (2010).

\section{ACKNOWLEDGEMENTS}

The authors would like to thank Kumaravel Kandaswamy for growing the biofilms used in Fig. 1 and Supplementary Fig. 8 and for performing the staining in Supplementary Fig. 8, Valerie Yong Fei Lee for growing the biofilms used in Fig. 5, Sujatha Subramoni for helping in the cloning and Sonia Monti for preparing the illustration in Fig. 1a. T.W. gratefully acknowledges funding from the Ministry of Education of Singapore (MOE2014T2-2-115). We acknowledge support by the National Research Foundation and Ministry of Education Singapore under its Research Centre of Excellence Programme.

\section{AUTHOR CONTRIBUTIONS}

T.W., S.A.R. and Y.C. conceived and designed the study. J.S., N.J.T.J.H. and B.K.P. performed the experiments and analysed the data. J.S., T.W. and S.A.R. wrote the manuscript.

\section{COMPETING INTERESTS}

The authors declare no competing interests.

\section{ADDITIONAL INFORMATION}

Supplementary information is available for this paper at https://doi.org/10.1038/ s41522-019-0107-4.

Correspondence and requests for materials should be addressed to S.A.R. or T.W.

Reprints and permission information is available at http://www.nature.com/ reprints

Publisher's note Springer Nature remains neutral with regard to jurisdictional claims in published maps and institutional affiliations.

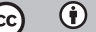

Open Access This article is licensed under a Creative Commons Attribution 4.0 International License, which permits use, sharing, adaptation, distribution and reproduction in any medium or format, as long as you give appropriate credit to the original author(s) and the source, provide a link to the Creative Commons license, and indicate if changes were made. The images or other third party material in this article are included in the article's Creative Commons license, unless indicated otherwise in a credit line to the material. If material is not included in the article's Creative Commons license and your intended use is not permitted by statutory regulation or exceeds the permitted use, you will need to obtain permission directly from the copyright holder. To view a copy of this license, visit http://creativecommons. org/licenses/by/4.0/

(c) The Author(s) 2019 